\title{
UNA NUEVA ESPECIE DE MALVASTRUM (MALVACEAE) DE LA RIOJA (ARGENTINA)
}

\author{
ANTONIO KRAPOVICKAS ${ }^{1} \dagger$
}

\begin{abstract}
Summary: Krapovickas, A. 2015. A new species of Malvastrum (Malvaceae) from La Rioja (Argentina). Bonplandia 24 (2): 77-80.
\end{abstract}

The new species Malvastrum multicuspidatum is here described. It is characterized by mericarps with 6-8 pairs of aristae instead of the 0-3 pairs as in the rest of the genus.

Key words: Argentina, Malvaceae, Malvastrum.

Resumen: Krapovickas, A. 2015. Una nueva especie de Malvastrum (Malvaceae) de La Rioja (Argentina). Bonplandia 24 (2): 77-80.

Se describe la especie Malvastrum multicuspidatum caracterizada por sus mericarpos con 6-8 pares de aristas dorsales, a diferencia del resto del género donde el número de aristas no pasa de tres.

Palabras clave: Argentina, Malvaceae, Malvastrum.

\section{Malvastrum sect. Multicuspidatum Krapov., sect. nov.}

Carpella matura 8-10, indehiscentia, multiaristata, aristae acutae.

Typus Malvastrum multicuspidatum Krapov.

\section{Malvastrum multicuspidatum Krapov. sp. nov.}

Fig. 1

Lamina late-ovata vel suborbicularia, supra et infra stellato pilosa. Floribus solitariis. Bracteolis 3, lanceolatis , $4 \mathrm{~mm}$ longae. Calyx $8 \mathrm{~mm}$ longus. Tubo stamineo $2 \mathrm{~mm}$ longus, subglabro. Styli 9-10. Carpidia $3 \mathrm{~mm}$ longa et 3,5 mm lata, hirsuta, apice aristatis, dorso 6-8 paria aristatis. Semina pilosa.
Typus: ARGENTINA. La Rioja: Dep. Coronel Felipe Varela, RN 40, a 39 km de Villa Unión en dirección a Nonogasta, 2926'14"S, 67'53'56"W, 1438 m s.m. 28-III-2012, J.D. Urdampilleta, F. Chiarini \& N. Moreno 630 (Holotypus CTES, isotypus CORD).

Pequeña mata de unos $20 \mathrm{~cm}$ alt. Tallo cubierto de pelos estrellados. Estípulas lineares, 4-5 $\mathrm{mm}$ long., estrellado-pilosas. Pecíolo 20-30 mm long. Lámina ovada, 2,5$3 \times 2,5-3 \mathrm{~cm}$, obtusa, suborbicular, margen apenas dentado, casi liso, ambas caras con pelos estrellados. Flores solitarias. Pedicelo $4 \mathrm{~mm}$ long., con pelos estrellados. Bractéolas 3, de $4 \mathrm{~mm}$ long., estrellado-pilosas. Cáliz 8 $\mathrm{mm}$ long., estrellado-piloso. Pétalos con la uña pilosa. Tubo estaminal $2 \mathrm{~mm}$ long., subglabro, con algunos pelos estrellados, anteras ca. 20.

${ }^{1}$ Instituto de Botánica del Nordeste IBONE (CONICET-UNNE, Facultad de Ciencias Agrarias), Sgto. Cabral 2131, C.C. 209, (3400) Corrientes, Argentina. 


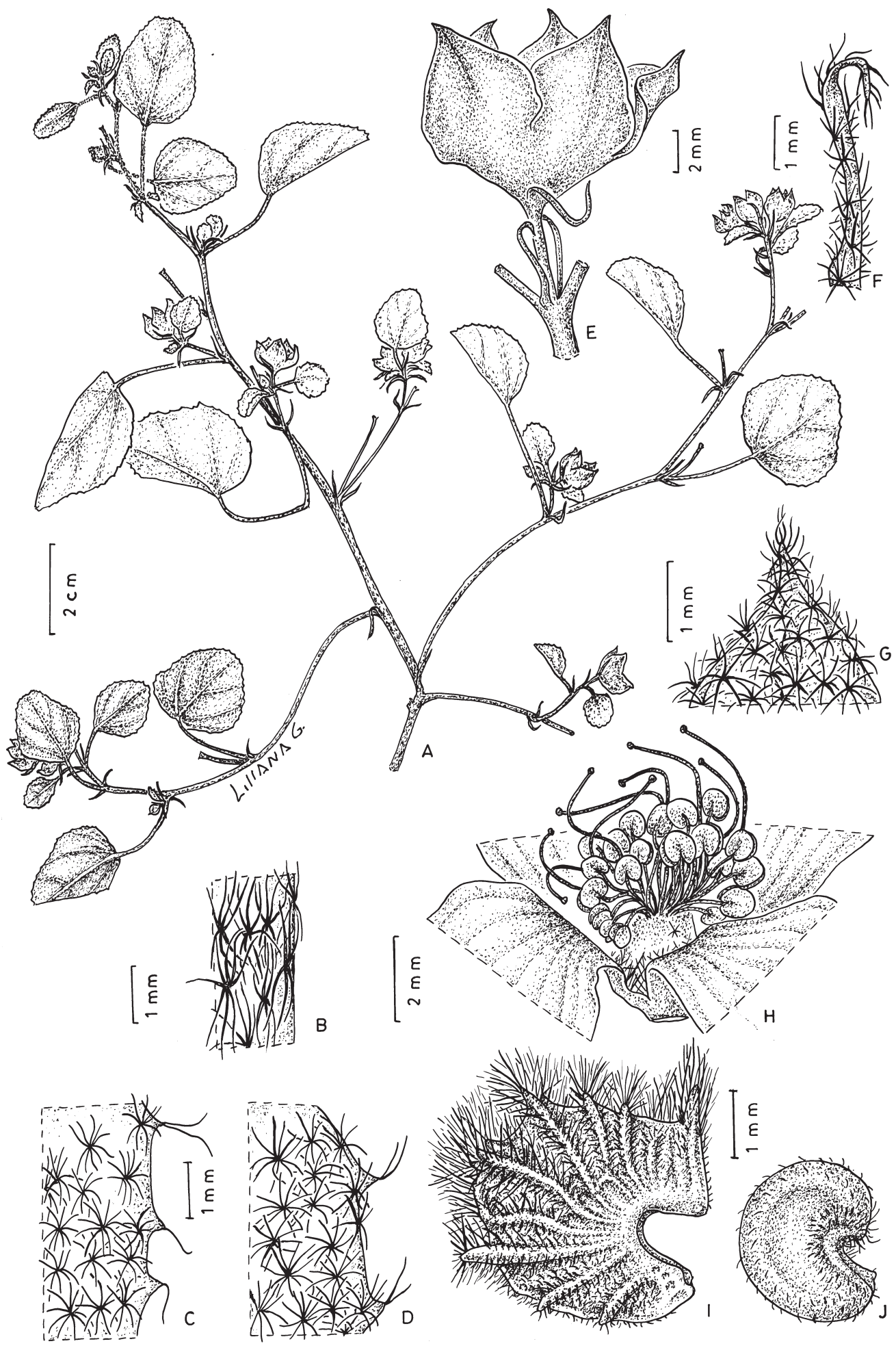

Fig. 1. Malvastrum multicuspidatum. A: Rama. B: Indumento del tallo. C: Indumento del epifilo. D: Indumento del hipofilo. E: Cáliz. F: Bractéola. G: Indumento del cáliz. H: Tubo estaminal. I: Mericarpo, vista lateral. J: Semilla (Urdampilleta et al.630, CTES). 
Estilos 8-10, estigmas en cabezuela. Mericarpos indehiscentes, $3 \times 3,5 \mathrm{~mm}$, hirsutos, con pelos simples hasta $1 \mathrm{~mm}$ long., con una arista apical y 6-8 pares de aristas dorsales correspondientes con las costillas de las caras laterales. Semilla solitaria, con pelos simples.

Distribución geográfica: Conocida sólo de la localidad tipo en el centro de la provincia de La Rioja, en el NW de la Argentina.

Obs. Malvastrum multicuspidatum se distingue del resto del género por su pequeño tamaño, por sus hojas suborbiculares levemente dentadas y principalmente por sus mericarpos pilosos multiaristados. En el resto del género el número de aristas no pasa de tres, como se puede apreciar en las ilustraciones de los mericarpos de todas las especies del género (Hill, 1982; Krapovickas, 1957, 1974, 2011).

Por sus mericarpos aristados, no alados y por la ausencia de brácteas bífidas en las flores M. multicuspidatum pertenece a la sección Multicuspidatum.

\section{Clave para reconocer las especies de Malvastrum de Argentina}

\section{Mericarpos aristados.}

2. Mericarpos con 8 pares de aristas. La Rioja.

(Secc. Multicuspidatum Krapov.)

M. multicuspidatum Krapov.

2'. Mericarpos uni-triaristados, aristas agudas.

3. Mericarpos no alados.

\section{(Secc. Coromandelianum Krapov.)}

4. Mericarpos triaristados.

5. Indumento del tallo constituído por pelos adpresos de 4 ramas paralelas.

6. Cáliz 9-12 mm long. Flores solitarias. Mericarpos con las caras laterales con pelos estrellados o simples pequeños y con pelos simples erectos en la cara superior. Chaco seco...

M. ionthacarpum Krapov.

6'. Cáliz 5-7 mm long. Mericarpos con las caras laterales glabras o con pelos estrellados punctiformes. M. coromandelianum (L.) Garcke

7. Flores solitarias o en glomérulos paucifloros hacia el ápice de las ramas. Cosmopolita. M. coromandelianum subsp. coromandelianum

7'. Flores en glomérulos axilares plurifloros, apretados. NW de Argentina y S de Bolivia. M. coromandelianum subsp. capitato-spicatum (Kuntze) S.R. Hill

5'. Indumento del tallo constituído principalmente por pelos simples, erectos, 1-2 $\mathrm{mm}$ long. y con muy pocos pelos de 4 ramas adpresos. NW de Argentina, S de Bolivia...... M. coromandelianum var. congestum R.E. Fr.

4'. Mericarpos con un apículo breve en el ápice. NE de Argentina.......... M. uniapiculatum Krapov. 3'. Mericarpos con los márgenes brevemente alados.

NW de Argentina y S de Bolivia

(Secc. Amblyphyllum S.R Hill)

'. Mericarpos lisos, no aristados.

8. Mericarpos sólo con pelos estrellados pequeños en la cara superior.

(Secc. Interruptum S.R. Hill)

9. Mericarpos 7-10, 1-1,5 × 1-1,5 mm. Estambres 5-7. $2 \mathrm{n}=12$. NW de Argentina, Bolivia. M. spiciflorum (Hassl.) Krapov.

9'. Mericarpos 10-12, 1,9-2 × 2-2,2 mm. Estambres 20-27. 2n=6. NW de Argentina y Bolivia. M. interruptum K. Schum.

8'. Mericarpos con pelos simples erectos en la cara superior.

(Secc. Americanum S.R. Hill).

$2 n=24$. Cosmopolita. M. americanum (L.) Torrey 


\section{Agradecimiento}

Agradezco a la Sra. Liliana Gómez la ilustración que acompaña esta descripción.

\section{Bibliografía}

HILL, S. R. 1982. A monograph of the genus Malvastrum
A.Gray (Malvaceae: Malveae). Rhodora 84: 1-83, 159-264, 317-409.

KRAPOVICKAS, A. 1957. Las especies de Malvastrum sect. Malvastrum de la flora Argentina. Lilloa 28: 181-195.

KRAPOVICKAS, A. 1974. Malvastrum grandiflorum, nueva especie de Malvácea de Bolivia. Bol. Soc. Argent. Bot. 15: 46-470.

KRAPOVICKAS, A. 2011. Novedades en el género Malvastrum (Malvaceae). Bonplandia 20: 55-72.

Original recibido el 27 de mayo de 2014; aceptado el 3 de diciembre de 2014. 